\title{
IMPROVING ACTIVE QUERIES WITH A LOCAL SEGMENTATION STEP AND APPLICATION TO LAND COVER CLASSIFICATION
}

\author{
S. Wuttke ${ }^{\mathrm{a}, \mathrm{b}, *}$, W. Middelmann ${ }^{\mathrm{a}}, \mathrm{U}$. Stilla ${ }^{\mathrm{b}}$ \\ a Fraunhofer IOSB, Gutleuthausstr. 1, 76275 Ettlingen, Germany - (sebastian.wuttke, wolfgang.middelmann)@iosb.fraunhofer.de \\ b Technische Universitaet Muenchen, Arcisstr. 21, 80333 Muenchen, Germany - stilla@tum.de
}

KEY WORDS: Active Learning, Remote Sensing, Land Cover Classification, Segmentation, Hierarchical Clustering, Active Queries

\begin{abstract}
:
Active queries is an active learning method used for classification of remote sensing images. It consists of three steps: hierarchical clustering, dendrogram division, and active label selection. The goal of active learning is to reduce the needed amount of labeled data while preserving classification accuracy. We propose to apply local segmentation as a new step preceding the hierarchical clustering. We are using the SLIC (simple linear iterative clustering) algorithm for dedicated image segmentation. This incorporates spatial knowledge which leads to an increased learning rate and reduces classification error. The proposed method is applied to six different areas of the Vaihingen dataset.
\end{abstract}

\section{INTRODUCTION}

Land cover is defined by the United Nations as "the observed (bio)physical cover on the earth's surface" (Di Gregorio and Jansen, 2000). To make informed political, economic, and social decisions it is important to know which land cover type is found in different areas of the earth (Anderson, 1976). Climate studies for example are in need of precise information about the distribution of different land cover types. In urban planning it is important to differentiate between closed and open soil to predict the effects of rainfall.

Today's constantly advancing remote sensing technology allows for higher resolutions, faster repetitions, and cheaper and therefore more ubiquitous sensors. The availability of smaller form factors enables the development of multi sensor platforms (Haraké et al., 2016). This leads to a manifold increase in available raw data. Processing these manually in a timely manner is impossible. To handle this challenge, advances in machine learning are necessary. To support this automation, two distinct information sources can be identified in remotely sensed data: spatial and spectral. The first is governed by the Smoothness Assumption (Schindler, 2012) which states that pixels have a higher probability of belonging to the same class if they are spatially closer. The second information source is governed by the Cluster Assumption (Patra and Bruzzone, 2011) which states that pixels have a higher probability of belonging to the same class if they are spectrally closer. These information sources are called redundancies by (Hasanzadeh and Kasaei, 2010). To exploit these information sources, unsupervised machine learning uses different segmentation and clustering techniques. This has the advantage that all available data can be used at once. The biggest disadvantage is that unsupervised techniques are hard to calibrate because of the missing explicit link between the unsupervised detected clusters and the classes of interest to the user (Fleming et al., 1975, Munoz-Mari et al., 2012, Lee and Crawford, 2004, Lee, 2004). In contrast, the supervised approach lets the user label instances with the desired class. This leads to improved classification results, but entails the additional costs of acquiring ground truth data. To gather these

\footnotetext{
${ }^{*}$ Corresponding author
}

in a remote sensing setting often requires expensive ground surveys or the use of experts in image interpretation. Reducing these costs by strategically choosing which label to acquire is goal of the active learning approach (Atlas et al., 1990, Settles, 2012). Active learning is investigated thoroughly on a theoretical level (Balcan et al., 2006, Hanneke, 2014, Kääriäinen, 2006) as well as applied in a variety of fields such as bio-medical (Cui et al., 2009, Pasolli and Melgani, 2010, Krempl et al., 2015) and image retrieval (Cheng and Wang, 2007, Liu et al., 2008, Zhang et al., 2008). A good overview of applied active learning in remote sensing is given in (Tuia et al., 2011, Bruzzone and Persello, 2010). Applications particular for land cover classifications can be found in (Demir et al., 2012). To combine these available tools in a manner that is computationally efficient, conserving human interaction time, and achieving high classification results, is a challenging task.

The principle of using a hierarchy to guide the sampling follows the work of (Dasgupta and Hsu, 2008). They start with a tree hierarchy and try to find the optimal pruning which corresponds to the optimal segmentation. It is applied to remote sensing in (Tuia et al., 2012) as the active queries method. They introduce strategies to select the optimal node for splitting and to choose the optimal leaf for querying the user. The result is a three stage approach: 1) create a hierarchical clustering, 2) find the optimal pruning, and 3) find the most informative sample and query its label.

This work is further extended in (Munoz-Mari et al., 2012) by establishing that incorporating spatial information increases the classification accuracy greatly. Our approach to incorporate spatial data is the use of a dedicated local segmentation algorithm as a preceding step. We chose the SLIC method (simple linear iterative clustering) by (Achanta et al., 2012) which restricts the grouping of pixels with a spatial term. Similar work is done in (Hasanzadeh and Kasaei, 2010) where they use principal component analysis and watershed transformation followed by a fuzzy c-means method and finally membership-connectedness based segmentation to retain spatially small regions. Spatial restricted segmentation is also done by (Senthilnath et al., 2012). They compare splitting by mean shift clustering, niche-particle swarm optimization, and glowworm swarm optimization. 


\begin{tabular}{|c|c|c|c|}
\hline Publication & $S$ & $\mathrm{C}$ & AL \\
\hline (Lee and Crawford, 2004) & $\otimes$ & $\otimes$ & $\square$ \\
\hline (Lee, 2004) & $\otimes$ & $\otimes$ & $\square$ \\
\hline (Lee and Crawford, 2005) & $\otimes$ & $\otimes$ & $\square$ \\
\hline (Marcal and Castro, 2005) & $\square$ & $\otimes$ & $\square$ \\
\hline (Bruzzone and Carlin, 2006) & $\otimes$ & $\otimes$ & \\
\hline (Dasgupta and Hsu, 2008) & $\square$ & $\otimes$ & $\otimes$ \\
\hline (Hasanzadeh and Kasaei, 2010) & $\otimes$ & $\square$ & $\square$ \\
\hline (Senthilnath et al., 2012) & $\otimes$ & $\square$ & \\
\hline (Tuia et al., 2012) & $\square$ & $\otimes$ & $\otimes$ \\
\hline (Munoz-Mari et al., 2012) & $\square$ & $\otimes$ & \\
\hline (Huo et al., 2015) & $\square$ & $\otimes$ & $\square$ \\
\hline This Work & $\otimes$ & $\otimes$ & $\otimes$ \\
\hline
\end{tabular}

Table 1. Overview of related work in comparison to the three main stages of the presented method: segmentation (S), clustering $(\mathrm{C})$, and active learning $(\mathrm{AL})$.

The clustering step in the work of (Tuia et al., 2012) creates a hierarchy which ignores spatial relationships and is based only on spectral information by using the bisecting $k$-means algorithm (Kashef and Kamel, 2009). Hierarchical global clustering is done in (Marcal and Castro, 2005) by using a linear combination of four indexes: Malahanobis distance, portion of shared boundary pixels, ratio of compactness, and amount of pixels in compared classes. Hierarchical clustering is also used in (Huo et al., 2015), but they use the hierarchy directly to influence a SVM by adapting its kernel through a linear combination of the RBF kernel and two hierarchy based similarity measures.

A multistage approach by combining the local segmentation and global clustering is presented in (Bruzzone and Carlin, 2006). They use hierarchical multilevel segmentation for context-driven feature extraction followed by SVM classification. A compound analysis for active learning in remote sensing is shown in (Wuttke et al., 2015). A two stage approach is also used in (Lee and Crawford, 2004, Lee, 2004): Local region growing segmentation by hierarchical clustering followed by global segmentation with a context-free similarity measure (Malahanobis distance). In the follow-up work (Lee and Crawford, 2005) Bayesian criteria are used in the segmentation step to separate regions with different characteristics and as a stopping rule for their global hierarchical clustering. Table 1 gives an overview of the related work.

The presented extension to the active queries method is the first work using a dedicated segmentation algorithm before creating a clustering hierarchy and applying active learning afterwards. This uniquely combines these three methods to classify remote sensing data into different land cover classes. The contributions of this paper are:

- Extend active queries with a dedicated segmentation step,

- Greatly increase the learning rate,

- Reduce the overall classification error.

The remainder of this paper is structured as follows. Section 2 details the individual steps of the proposed method. Section 3 describes the used remote sensing dataset and the setup of the conducted experiments. Section 4 discusses the results of the experiments and compares the different approaches. Section 5 draws the conclusion of this paper.

\section{PROPOSED METHOD: ACTIVE QUERIES WITH LOCAL SEGMENTATION}

The presented extension to active queries adds a new step before the creation of the hierarchy. This approach has the advantage that subsequent steps do not need to be changed. The resulting four step method is described in this section.

\subsection{Local segmentation}

Incorporating spatial features reduces the classification error significantly, (Munoz-Mari et al., 2012). They do this by using two types of spatial information:

1. standard morphological opening and closing operations;

2. normalized latitude and longitude coordinate vectors.

We take an alternative approach to incorporate spatial knowledge. We propose the use of a dedicated local segmentation step which combines individual pixels into groups based on a distance measure. The term "local" denotes that the distance measure takes also the spatial distance into account and not just spectral features. This step is applied to the whole image.

The segmentation algorithm can be chosen from a wide range of available methods. One example are graph based methods like in (Wassenberg et al., 2009). Though at this stage it is important that, as mentioned above, spatial features are used. We chose the SLIC (simple linear iterative clustering) algorithm because it is fast, has a single parameter version, is deterministic, and has improved segmentation performance compared to other state of the art methods (Achanta et al., 2012).

The SLIC algorithm is a superpixel based segmentation method that uses localized $k$-means clustering. Its single parameter $k$ is the desired number of approximately equally sized superpixels. The image (containing $N$ pixels) is seeded with $k$ cluster centers $C_{i}$ on a regular grid, spaced $S=\sqrt{N / k}$ pixels apart. The color image is transformed into the CIELAB color space $\left[l^{*} \times a^{*} \times b^{*}\right]$ as specified by the International Commission on Illumination (French Commission internationale de l'éclairage). Here $l^{*}$ is the lightness $\left(l^{*}=0\right.$ equals black, $l^{*}=100$ equals white), $a^{*}$ is the position between red/magenta and green $\left(a^{*}<0\right.$ are green values, $a^{*}>0$ are magenta values), and $b^{*}$ is the position between yellow and blue $\left(b^{*}<0\right.$ are blue values, $b^{*}>0$ are yellow values). The spatial dimensions $(x, y)$ are concatenated so that each $C_{i}$ is a point in $\left[l^{*} \times a^{*} \times b^{*} \times x \times y\right]$ which is the Cartesian product of all transformed spectral and spatial dimensions. In the assignment step each pixel is associated to the nearest cluster center. The key optimization is that only pixels in a region of size $2 S \times 2 S$ are considered instead of the whole image. This reduces the search complexity from $O(k N I)(I$ being the number of SLIC iterations until convergence) to $O(N)$, see (Achanta et al., 2012) for details.

Next, in the update step, each cluster center $C_{i}$ is adjusted to the mean of all pixels assigned to that cluster using the $L^{2}$ norm. Ten iterations of both steps suffice for most images (Achanta et al., 2012). In a post-processing step disjoint pixels are assigned to nearby superpixels. The distance measure used in the assignment step is:

$$
D=\sqrt{d_{c}^{2}+\left(\frac{d_{s}}{S}\right)^{2} m^{2}} .
$$


where:

$$
\begin{aligned}
& d_{c}=\text { Euclidean distance of color components } \\
& d_{s}=\text { Euclidean distance of spatial components } \\
& m=\text { scaling parameter }
\end{aligned}
$$

The parameter $m$ is used to scale the color distance as well as to weigh the importance of the spatial term.

We propose combining this spatially aware segmentation with the smoothness assumption, which states that neighboring pixels have a high probability of belonging to the same class. Therefore each segment can be represented by a single feature vector without losing too much information. The simplest method is to take the average spectrum of all pixels belonging to one segment. This leads to the biggest advantage of this approach: increased robustness by averaging out multiple spectra which reduces noise and removes outliers. The result of this step are "representative" pixels which can be used further without the need to modify the subsequent steps. If the data has lots of outliers simply taking the average can lead to errors. Other possibilities are to remove outliers before calculating the average, using the median instead of the mean, or filtering based on an assumed normal distribution.

\subsection{Clustering hierarchy}

Clustering is similar to segmentation as it also groups elements together guided by a distance measure. The difference in this step is that the groups are nested and a hierarchy is formed. Whereas in the first step the segmentation is a flat partitioning of the image.

To generate the clustering hierarchy the bisecting $k$-means algorithm is used. Initially, the whole dataset is contained in one cluster, which forms the root of the resulting binary tree hierarchy. In each iteration the largest cluster is chosen and bisected $(k=2)$. The two resulting clusters are added as children of the former cluster in the hierarchy. This is repeated until each cluster contains only one element or until a fixed number $B$ of bisections is reached; (Munoz-Mari et al., 2012) use $B=4096$. The distance measure used is the cosine distance $d\left(x_{i}, x_{j}\right)=$ $x_{i}^{\top} x_{j} /\left\|x_{i}\right\|\left\|x_{j}\right\|$.

\subsection{Finding the optimal pruning}

After the clustering step is completed the binary tree represents different clusterings of the image. Each node $v$ of the tree has a probability that all its elements belong to class $w$. Following (Tuia et al., 2012) it can be estimated by $p_{v, w}=l_{v, w} / n_{v}$, where $l_{v, w}$ is the number of labeled elements and $n_{v}$ is the total number of elements in node $v$. If only a few labels are known, this probability is very uncertain. We use the definitions from (MunozMari et al., 2012) for the lower $(L B)$ and upper $(U B)$ confidence bounds:

$$
\begin{array}{r}
p_{v, w}^{L B}=\max \left(p_{v, w}-\Delta_{v, w}, 0\right) \\
p_{v, w}^{U B}=\min \left(p_{v, w}+\Delta_{v, w}, 1\right)
\end{array}
$$

where:

$$
\begin{aligned}
& \Delta_{v, w}=\left(c_{v} / n_{v}\right)+\sqrt{c_{v} p_{v, w}\left(1-p_{v, w}\right) / n_{v}} \\
& c_{v}=1-\left(l_{v} / n_{v}\right)
\end{aligned}
$$

The confidence term $\Delta_{v, w}$ includes a correction factor $c_{v}$ which is proportional to the number of elements in $v$. Assigning the label $w$ to node $v$ is very certain if the lower confidence bound of class $w$ is at least twice as high as the upper confidence bound of the second most probable class $w^{\prime}$. This is called an admissible labeling:

$$
p_{v, w}^{L B}>2 p_{v, w^{\prime}}^{U B}-1 \quad \forall w^{\prime} \neq w .
$$

The estimated error when assigning label $w$ to the node $v$ can be calculated:

$$
\tilde{\epsilon}_{v, w}= \begin{cases}1-p_{v, w}, & \text { if }(v, w) \text { is admissible } \\ 1, & \text { otherwise }\end{cases}
$$

A cut through the tree removes (prunes) lower nodes and creates a subtree above the cut. If the leafs of the subtree contain all elements of the dataset the pruning is called complete. The optimal pruning is a complete pruning which results in the lowest estimated classification error. The overall error of a pruning is reduced by splitting a node if the sum of the error of its children is lower, see (Dasgupta and Hsu, 2008) for a proof. Therefore the pruning algorithm splits node $v$ into its children $l$ and $r$ if

$$
\tilde{\epsilon}_{v, w}>\tilde{\epsilon}_{v_{l}, w_{l}}+\tilde{\epsilon}_{v_{r}, w_{r}}
$$

A property of this approach worth noting is that for each iteration the optimal pruning forms a partition of the whole image which induces a complete classification. Therefore the process can be stopped at any time to receive a valid result.

\subsection{Active sampling}

The previous step finds the optimal pruning which induces the lowest estimated classification error. To improve this more labeling information is needed. A naive approach would be to choose a random element and query the label from the user. This leads certainly to an increase in classification quality, but needs a lot of queries and human interaction time. By using active learning methods this effort can be reduced by up to $50 \%$ (Munoz-Mari et al., 2012).

To achieve this, the element which lowers the classification error the most must be identified. The employed active querying strategy consists of two sub-strategies:

1. $s_{i}$ : selecting the best node,

2. $d_{i}$ : descending to the best leaf.

The first part identifies the node of the current pruning which would profit the most from getting new label information. The second part descends from the selected node iteratively either into the left or the right child until a leaf is reached. Different measures for both strategies are possible. They are used to calculate probabilities with which the node is chosen. Not using fixed decisions allows the method to make small mistakes and discover new clusters. Based on these a leaf is chosen. A basic strategy is a probability proportional to the node size $s_{0}=d_{0}=n_{v}$. This is equivalent to random sampling. An active strategy weighs the size with the node uncertainty $s_{1}=d_{1}=1-p_{v, w}^{L B}$. A strategy considering only the $k$ nodes which maximize the $s_{1}$ value is $s_{2}=n_{v^{k}}\left(1-p_{v^{k}, w}^{L B}\right)$. A summary of the different strategies is given in Table 2.

If the selected leaf contains multiple elements, one is chosen at random. In the case of the original version from Tuia and MuñozMarì such an element is a single pixel. In the proposed extension 


\begin{tabular}{lccc}
\hline & Select & \multicolumn{2}{c}{ Descent } \\
\hline$s_{0}:$ & $n_{v}$ & $d_{0}:$ & $n_{v}$ \\
$s_{1}:$ & $n_{v}\left(1-p_{v, w}^{L B}\right)$ & $d_{1}:$ & $n_{v}\left(1-p_{v, w}^{L B}\right)$ \\
$s_{2}:$ & $n_{v^{k}}\left(1-p_{v^{k}, w}^{L B}\right)$ & & \\
\hline
\end{tabular}

Table 2. Different strategies for calculating probabilities to select nodes and descend to leafs. $s_{0}$ and $d_{0}$ are equivalent to random sampling, the others are active learning strategies.

it is instead a whole segment. This is an advantage because it reduces the effort for the user additionally to the reduction gained by the active learning approach. The label probabilities, confidence bounds, and estimated errors in the hierarchy are updated to integrate the new information. Finding the best pruning based on the new values starts the next iteration.

\section{EXPERIMENTAL SETUP AND DATASETS}

Our proposed extension was tested with different experiments and on different datasets. This sections details the experimental setup and presents the used datasets.

\subsection{Experimental setup}

To test our approach we conducted five experiments. For every experiment the size of the classification error is tracked during the execution of the algorithm and plotted over the number of queried samples. The resulting graph is called a learning curve. It can be used to compare the efficiency of different methods and parameter settings. Because some of the steps are based on randomization, we repeated every experiment 10 times and report the mean and standard deviation. To ensure comparability we used the same definition of classification error as (Munoz-Mari et al., 2012). Each experiment varied only in one parameter, every other parameter was kept constant. Table 3 gives an overview about the used parameters. The five experiments are:

1. Reproducing results of (Munoz-Mari et al., 2012)

2. Removing outliers

3. Removing border class

4. Number of bisections

5. Different datasets

3.1.1 Reproducing Devis Tuia, a co-author of (Munoz-Mari et al., 2012), provided us with the MATLAB code of their active query implementation. We applied the original method on the Brutisellen dataset to confirm that the code works as intended. Afterwards we applied their method on the Vaihingen dataset to get a baseline to compare with our proposed extension. The most successful strategy was selected and applied to the following experiments.

3.1.2 Removing outliers As described in the section about segmentation (2.1) the calculation of the representative pixel can lead to errors if the segments contain many outliers. We therefore removed a varying fraction $\alpha$ of pixels and recalculated the mean afterwards. The pixels removed are the ones with the largest distance to the mean of all pixels. The chosen fractions are $\alpha=$ $\{0,0.1,0.25,0.4\}$.
3.1.3 Number of bisections The number of bisections $B$ in the hierarchical clustering step influences the amount of nodes in the tree. By choosing a lower number the execution time is faster, because fewer nodes need to be considered in the pruning and active sampling steps. By choosing a higher number the clustering fits better to the data, so that fewer segments get falsely combined. To test this trade-off, we set the number of bisections to $B=\{2500,5000,7500\}$.

3.1.4 Removing border class Pixels that are at the border of class segments often contain spectral information from multiple classes. Instead of removing outliers as in experiment 2, here we ignore the complete class of border pixels. This is purely for academic reasons since in a production system the ground truth information of which pixels belong to the border class is not known.

We supply the original method with the raw pixel data from the full image. This version is called "Pixel". For comparison we apply the proposed local segmentation and execute the method on the representative pixels. This version is called "SLIC". We report the resulting learning curves of the Pixel and SLIC versions with the variations "w/ border" and "w/o border".

3.1.5 Different datasets The main experiment is the comparison of the original method and our extension on different datasets. We chose a subset of six areas from the Vaihingen dataset that are well distributed and contain different characteristics (amount of residential/industrial/vegetation) of the whole dataset.

\subsection{Datasets}

The ISPRS Benchmark Test on Urban Object Detection and Reconstruction ${ }^{1}$ introduced the Vaihingen dataset and made it publicly available. It contains 33 aerial images of the town of Vaihingen in Baden-Württemberg, Germany (see Figure 1). Each image has a resolution of roughly 2,000 by 2,000 pixels and a ground sampling distance (GSD) of $9 \mathrm{~cm}$. For each pixel there are intensity values for three channels: near infrared, red, and green. Height information acquired by a LiDAR scanner is also available, but not used in this work. Ground truth is provided for 16 areas and has five classes (Car, Tree, Low vegetation, Building, Impervious surfaces). Additionally there is a rejection class (Clutter / Background) which captures pixels not belonging to any of the five main classes so that every pixel has a ground truth value. A second set of ground truth data contains a 3 pixels wide border between sections of different classes. The reasoning being that those pixels, in areas where two materials meet, very likely contain spectra from both. Those pixels can interrupt the learning process. To handle these separately, they form a sixth class: border. Table 4 gives an overview of all chosen areas. For completeness the Brutisellen dataset used in (Munoz-Mari et al., 2012) is included.

\section{RESULTS AND DISCUSSION}

This section reports and discusses the results of the experiments presented in the previous section.

\footnotetext{
${ }^{1}$ The Vaihingen data set was provided by the German Society for Photogrammetry, Remote Sensing and Geoinformation (DGPF) (Cramer, 2010): www.ifp.uni-stuttgart.de/dgpf/DKEP-Allg.html.
} 


\begin{tabular}{ccccl}
\hline Experiment & $\alpha$ & Border & $B$ & Dataset \\
\hline 1 & - & - & - & Brutisellen, Vaihingen \#7 \\
2 & $\{0,0.1,0.25,0.4\}$ & w/ & 5000 & Vaihingen \#7 \\
3 & 0.25 & $\{$ w/, w/o $\}$ & 5000 & Vaihingen \#7 \\
4 & 0.25 & w/ & $\{2500,5000,7500\}$ & Vaihingen \#7 \\
5 & 0.25 & w/ & 5000 & Vaihingen \#\{5, 7, 15, 23, 30, 37\} \\
\hline
\end{tabular}

Table 3. Overview of the different parameter variations for the five experiments. Experiments 1-4 were also executed on the other selected areas of the Vaihingen dataset with very similar results. Therefore only area \#7 is reported.

\begin{tabular}{|c|c|c|c|c|c|c|}
\hline Dataset & Sensor & $\begin{array}{l}\text { Spectral } \\
\text { bands }\end{array}$ & $\mathrm{GSD}[\mathrm{m}]$ & Pixel count & Classes & Class distribution \\
\hline Brutisellen & QuickBird & 4 & 2.40 & 40,762 & 9 & \\
\hline Vaihingen (\#5) & Intergraph/ZI DMC & 3 & 0.09 & $4,825,059$ & 6 & \\
\hline Vaihingen (\#7) & Intergraph/ZI DMC & 3 & 0.09 & $4,825,059$ & 6 & \\
\hline Vaihingen (\#15) & Intergraph/ZI DMC & 3 & 0.09 & $4,916,019$ & 6 & \\
\hline Vaihingen (\#23) & Intergraph/ZI DMC & 3 & 0.09 & $4,838,646$ & 6 & \\
\hline Vaihingen (\#30) & Intergraph/ZI DMC & 3 & 0.09 & $4,956,842$ & 6 & \\
\hline Vaihingen (\#37) & Intergraph/ZI DMC & 3 & 0.09 & $3,982,020$ & 6 & \\
\hline
\end{tabular}

Table 4. Overview of the different datasets. All datasets are aerial images of urban areas. Pixel counts are without background. Classes in Brutisellen: residential, commercial, vegetation, soil, bare soil, roads, pools, parkings, vegetation2. Classes in Vaihingen: impervious surfaces, building, low vegetation, tree, car, border.

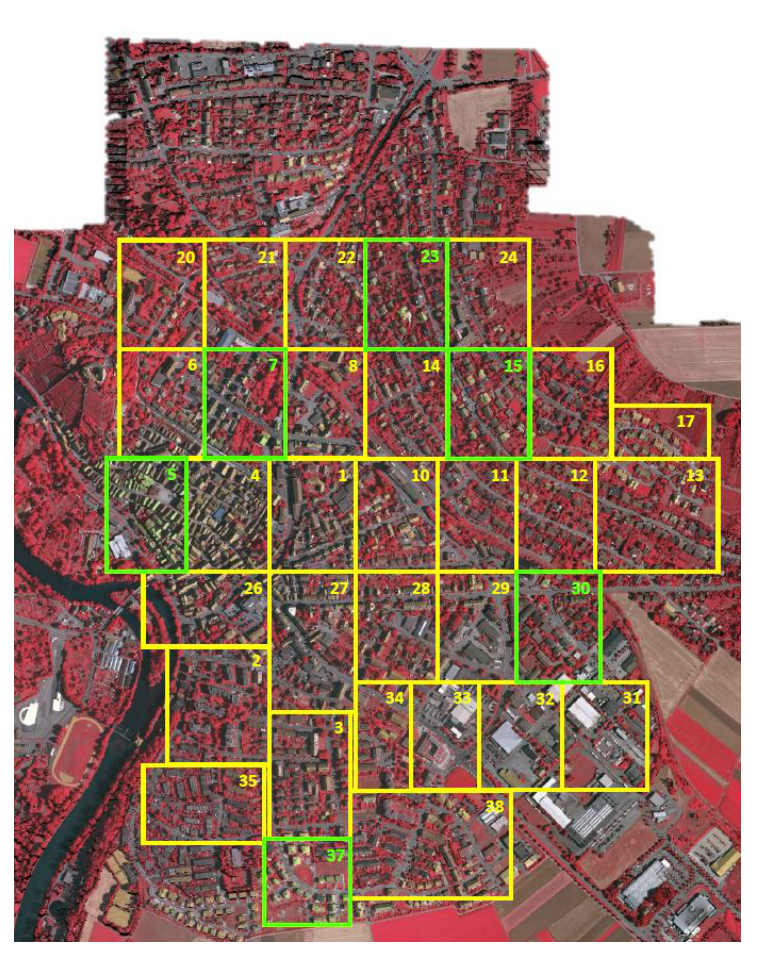

Figure 1. Overview of all 33 tiles of the Vaihingen dataset. Marked in green are the areas chosen for this work. Criteria for the choice were: ground truth available, distributed over whole area, representing different characteristics from the whole dataset (ratio of residential/industrial/vegetation).

\subsection{Reproducing}

We were able to reproduce the results from (Munoz-Mari et al., 2012) for the Brutisellen dataset. Though we arrived at a final error of $12 \%$ instead of $8 \%$, we observed the same effects regarding the effectiveness of the active learning strategies, see Figure 2(a). These improve the learning rate and lead to a lower overall classification error compared to random sampling strategies. Applying the same method to area 7 of the Vaihingen dataset leads to less definitive results, see Figure 2(b). Active learning still outperforms the random sampling strategies, but by a smaller margin. This is a sign that the classification of this dataset is much more challenging. The main reason is that it has only three spectral bands (which is $25 \%$ fewer than Brutisellen). Another source for complications could be the much higher spatial resolution of this dataset because this requires a different weighting between spectral and spatial features. Furthermore the number of pixels in the Vaihingen dataset is 100 times greater. If the fixed number of bisecting iterations leads to an inadequate granularity of the clustering hierarchy is tested in experiment 3 (see 4.4 ).

As reported in (Munoz-Mari et al., 2012) the active strategies $s_{1}, s_{2}, d_{1}$ outperform the random strategies $s_{0}, d_{0}$ because they actively focus on the most uncertain leafs. On the Vaihingen dataset strategy $\left(s_{1}, d_{1}\right)$ delivered slightly more consistent results and is therefore chosen for the remainder of the experiments.

\subsection{Removing outliers}

Varying the fraction of pixels that are removed before averaging the spectra to create the representative pixel, has an effect of $5 \%$ on the classification error (equals about one percent point), 


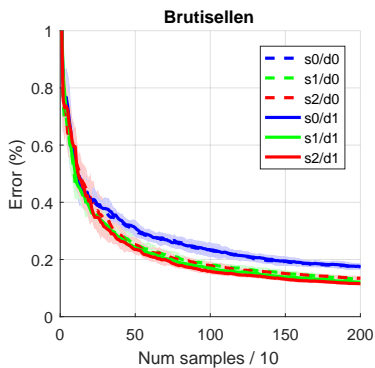

(a)

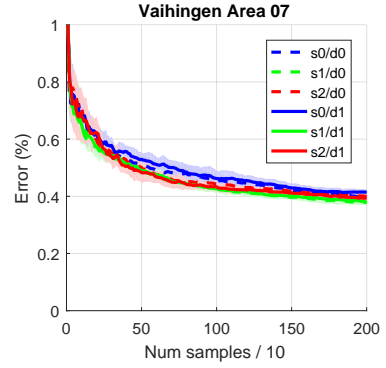

(b)

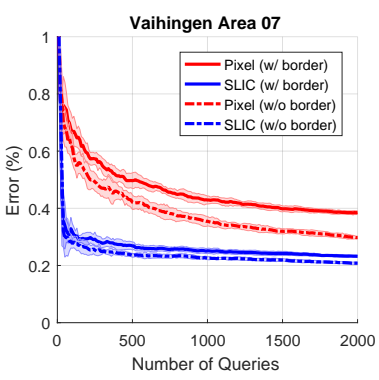

(a)

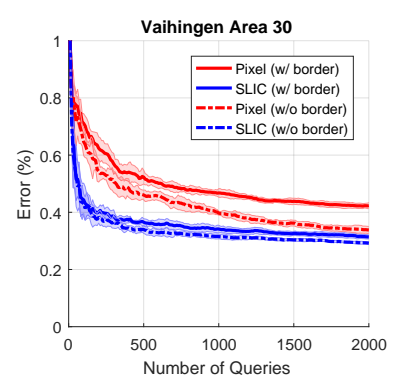

(b)
Figure 2. Reproduction of the results from (Munoz-Mari et al., 2012) for the Brutisellen dataset (a). The result is the same as originally reported, though the final classification error was $12 \%$ in our case instead of $8 \%$. The active strategies s1 and s2 clearly outperform the random sampling strategy s0. Applied to area 7 of the Vaihingen dataset, the results are not as distinct, but active learning is still better (b).

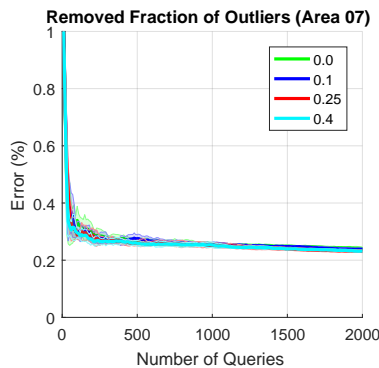

(a)

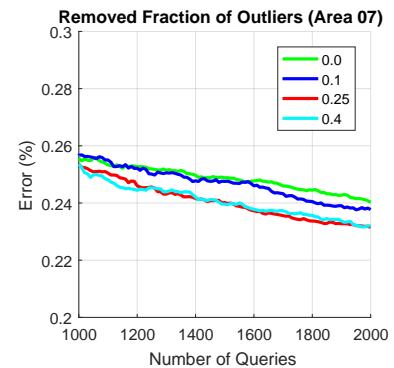

(b)
Figure 3. Removing varying fractions of pixels that are considered outliers (largest distance to the mean) has a small but noticeable effect on the classification error. The right figure is a closeup of later iterations. The standard deviations are not shown to provide a less cluttered image.

see Figure 3. This is a sign that the segments contain some outliers which negatively affect the mean. But removing more than $25 \%$ of the pixels does not affect the classification error further. Other possibilities of removing outliers can be considered in future work. For example using the median, removing outliers based on pixels with high gradients, or assuming a normal distribution and a cutoff threshold based on its standard deviation. After evaluating these results we chose a moderate amount of $\alpha=0.25$ to remove.

\subsection{Removing border class}

The results are an almost halved classification error in area 7 and $25 \%$ reduced error in area 30, see Figure 4 . The red curves are the original pixel based method, the blue curves are our proposed method based on the SLIC segmentation. The solid lines show the results of including border pixels whereas the dashed lines are from excluding them before clustering. The main contributing factor is the handling of mixed pixels by our method. This is also the reason why our method does not profit as much as the original method from removing the class of border pixels beforehand. The learning curve of the Pixel w/o border case is decreasing faster than the case Pixel w/ border, but not reaching the quality of the SLIC versions. This suggests that there is less noise in the clustering (it is lower than the w/ border case), but much information is "hidden" in the leafs of the tree.
Figure 4. Results of extending active queries with the proposed local segmentation for area 7 (a) and 30 (b) of the Vaihingen dataset. The red learning curves ("Pixel") are achieved by applying the original active query method directly on the pixels of the image. The blue curves ("SLIC") are achieved when the proposed extension is used with the SLIC algorithm. The dashed lines are the results of removing mixed pixels from the border regions beforehand.

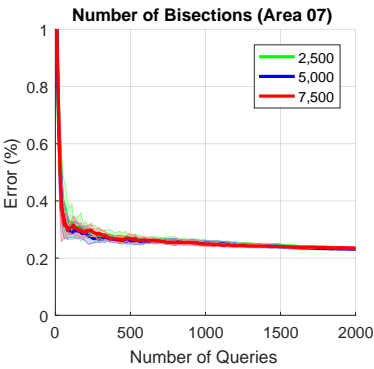

(a)

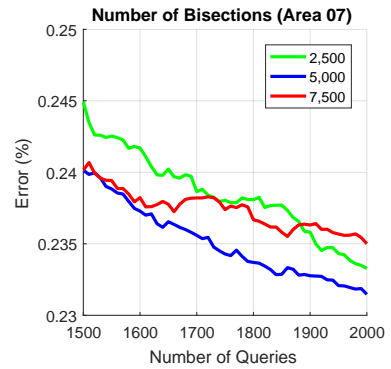

(b)
Figure 5. Varying the number of bisections in the hierarchical clustering step leads to no significant change (less than 0.4

\subsection{Number of bisections}

The number of bisecting iterations in the clustering step is limited and remaining elements are grouped together in the leafs of the tree. Increasing the number of bisections leads to a deeper tree which increases the computation time for all following steps, yet there is no significant effect (less than $1 \%$ or 0.4 percent points) on the classification error, see Figure 5.

A binary tree created by $B$ bisections has $B+1$ leafs. Choosing the number of SLIC superpixels $k$ greatly larger than $B$ therefore just leads to more segments grouped together within the leafs, where they do not have an effect on the active sampling strategy. To account for those segments a third querying strategy (see 2.4), for deciding which segment to pick from within a leaf, is needed. This is out of the scope of this paper, but is interesting for future work. We chose $k=10,000$ which leads to two segments per leaf on average and visually convincing segmentation results (see Figure 6). This choice leads to an oversegmentation. This is favorable since the clustering step can handle combining segments, but not splitting them if they were joined too soon. The result is a reduction of the number of elements to be clustered by a factor of 500. This leads to a faster clustering step, but comes with the added execution time of the new segmentation step. Overall, the computational complexity does not change since both steps use variations of the $k$-means clustering algorithm. percent points) in classification error. 


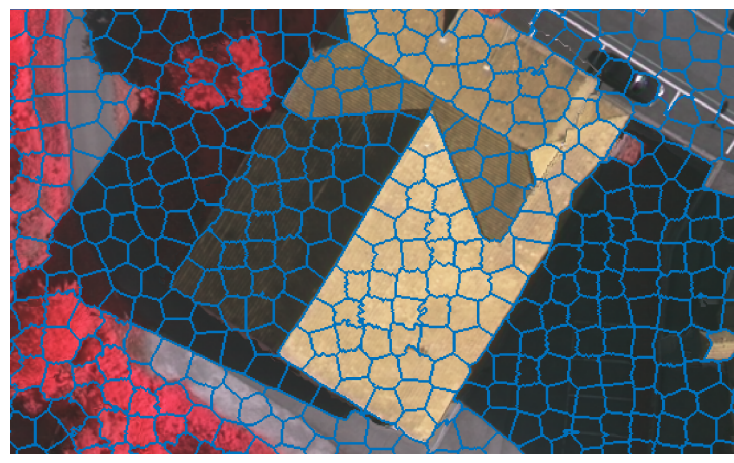

Figure 6. Detail of the SLIC segmentation result. The oversegmentation is advantageous because the segments can be grouped together later in the clustering step, but undersegmentation cannot be corrected.

\subsection{Different datasets}

The results on the different datasets are very similar, see Figure 7. All show a large reduction of the classification error if the proposed method is used. This improvement is based on the removing of mixed pixels which otherwise would lead to a clustering inconsistent with the ground truth. This cannot be fixed by labeling more samples and is the reason the classification error does not improve much further after 1,000 queries are reached. With the proposed method on the other hand, the clustering fits much better to the ground truth. Then even a few labeled samples are enough to reach a low classification error.

The use of the active learning strategy $\left(s_{1}, d_{1}\right)$ also improves the classification error compared to random sampling, but has a lot less influence than the use of the additional segmentation step. The reason for this is that active learning cannot compensate for noise in the clustering. This is a disadvantage of the proposed method. A solution could be an update of the clustering hierarchy after more information, by querying labels from the user, is available.

\subsection{General results}

Visualizing the specific samples that were queried is a valuable tool in analyzing active learning algorithms. Since there is only a minor difference between active and random sampling in the presented method, this tool cannot be applied here. The selection patterns are visually indistinguishable and therefore are not reported here.

The resulting classification maps at different stages of the learning process are displayed in Figure 8. In early stages (100 queries) not all classes are discovered. This is a sign of missing diversity during the selection process. This should be addressed by incorporating a penalty for querying in already known areas into the selection strategies. After 500 queries there are not many changes observable which matches the learning curves in Figure 7.

\section{CONCLUSION}

This paper presented an extension to the active queries method by adding a new first step of local segmentation. The results were a drop in the overall classification error and an increased learning rate that is reaching certain classification quality with fewer labeled samples. This was achieved by replacing the segments generated by the SLIC algorithm with representative pixels. The cause of this effect is that mixed pixels are inherently

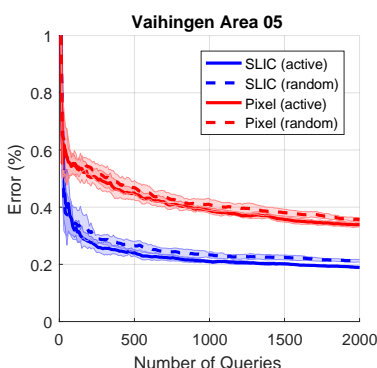

(a)

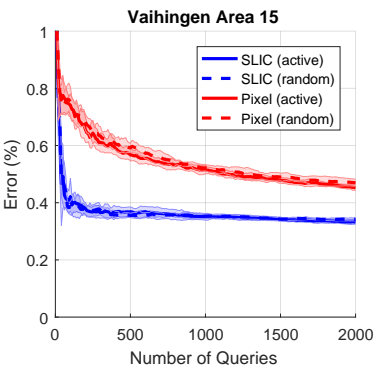

(c)

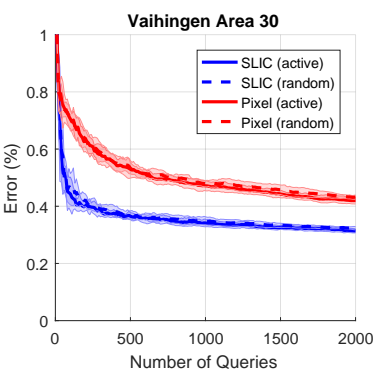

(e)

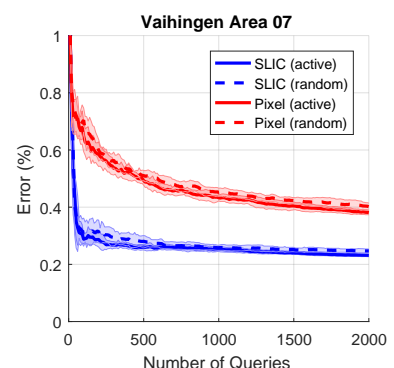

(b)

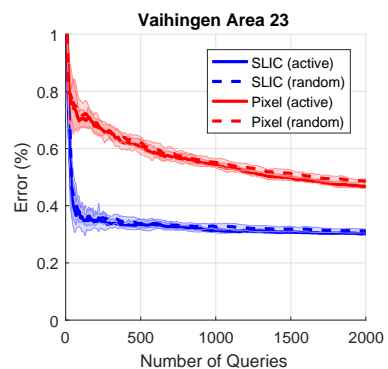

(d)

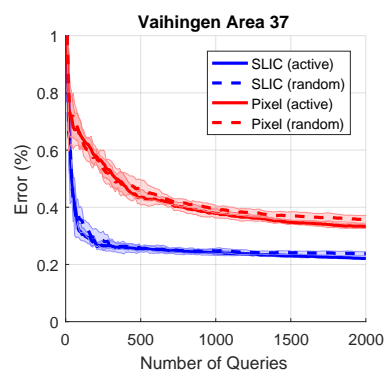

(f)
Figure 7. Comparison of the original pixel based active queries method (red) and the presented extension based on the SLIC algorithm (blue). Also shown is a comparison between random sampling (dashed lines) and active learning (solid lines). Both active learning and the handling of mixed pixels improve the classification error, but the extraction of representative pixels far outweighs the effects of the active learning. 


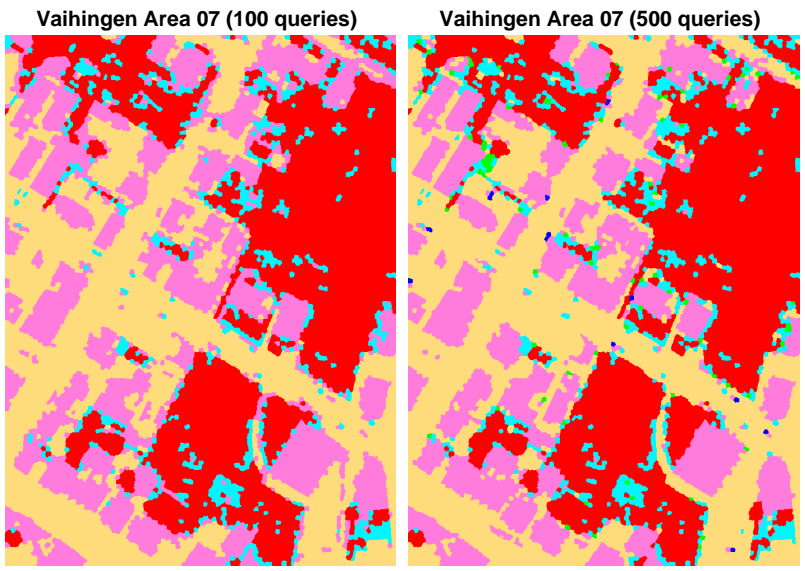

Vaihingen Area 07 (1000 queries)
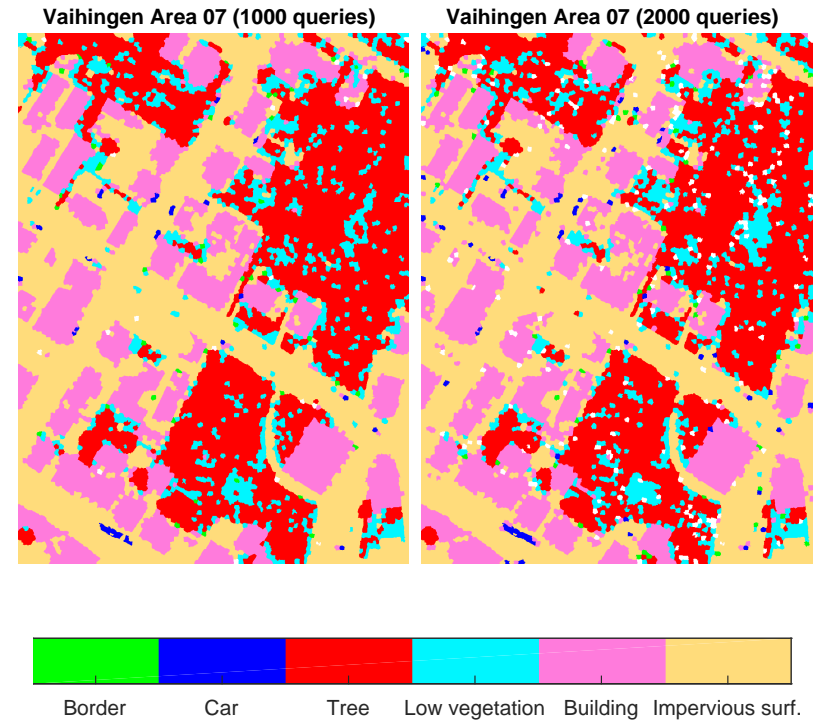

Figure 8. Classification maps at different stages of the learning process. At 100 queries not all classes are discovered. At 500 queries all classes are present and the result looks much like the final result. Between 1,000 and 2,000 queries are not many changes which matches the observations from the learning curves. uncertain and hinder the learning process. Therefore, removing them speeds up the learning rate. A similar, but less pronounced, effect was achieved by removing mixed pixels from the dataset entirely. This was possible because the Vaihingen dataset provides suitable ground truth. Since not all datasets offer this, the proposed extension is a helpful tool in handling mixed pixels in remote sensing data.

Our experiences with the SLIC algorithm show that it is very easy to handle. An advantage is the deterministic behavior, which ensures reproducible results. The regular structure of the seed points and missing merging of segments lead to oversegmentation. In the presented method this is beneficial, but in other contexts it could be a downside. To use it with hyperspectral images, either its combined Euclidean distance measure needs to be extended or a preprocessing step to mitigate nonlinearities like in (Gross et al., 2015) can be applied.

The bisecting $k$-means algorithm is a very good choice because it leads to a natural hierarchical clustering of the data. A downside is the random initialization. This requires repeated execution to get reliable results. A deterministic variant might be easier to study and lead to more insights.

Overall, the active queries method is a very beneficial tool to incorporate active learning into remote sensing classification tasks. It is easily adaptable to different strategies and extensions.

\section{REFERENCES}

Achanta, R., Shaji, A., Smith, K., Lucchi, A., Fua, P. and Susstrunk, S., 2012. Slic superpixels compared to state-of-theart superpixel methods. IEEE Transactions on Pattern Analysis and Machine Intelligence 34(11), pp. 2274-2282.

Anderson, J. R., 1976. A Land Use and Land Cover Classification System for Use with Remote Sensor Data. Geological Survey professional paper, U.S. Government Printing Office.

Atlas, L. E., Cohn, D. and Ladner, R., 1990. Training connectionist networks with queries and selective sampling. In: Advances in Neural Information Processing Systems, Morgan Kaufmann, San Mateo, Calif, pp. 566-573.

Balcan, M. F., Beygelzimer, A. and Langford, J., 2006. Agnostic active learning. In: International Conference on Machine Learning, pp. 65-72.

Bruzzone, L. and Carlin, L., 2006. A multilevel context-based system for classification of very high spatial resolution images. IEEE Transactions on Geoscience and Remote Sensing 44(9), pp. 2587-2600.

Bruzzone, L. and Persello, C., 2010. Recent trends in classification of remote sensing data: active and semisupervised machine learning paradigms. In: International Geoscience and Remote Sensing Symposium, IEEE, pp. 3720-3723.

Cheng, J. and Wang, K., 2007. Active learning for image retrieval with co-svm. Pattern Recognition 40(1), pp. 330-334.

Cramer, M., 2010. The dgpf-test on digital airborne camera evaluation overview and test design. PFG Photogrammetrie, Fernerkundung, Geoinformation 2010(2), pp. 73-82. 
Cui, B., Lin, H. and Yang, Z., 2009. Uncertainty sampling-based active learning for protein-protein interaction extraction from biomedical literature. Expert Systems with Applications 36(7), pp. 10344-10350.

Dasgupta, S. and Hsu, D. J., 2008. Hierarchical sampling for active learning. In: International Conference on Machine Learning, pp. 208-215.

Demir, B., Bovolo, F. and Bruzzone, L., 2012. Detection of landcover transitions in multitemporal remote sensing images with active-learning-based compound classification. IEEE Transactions on Geoscience and Remote Sensing 50(5), pp. 1930-1941.

Di Gregorio, A. and Jansen, L. J. M., 2000. Land cover classification systems (LCCS): Classification concepts and user manual. Food and Agriculture Organization of the United Nations, Rome.

Fleming, M. D., Berkebile, J. S. and Hoffer, R. M., 1975. Computer-aided analysis of landsat-1 mss data: A comparison of three approaches, including a 'modified clustering' approach. Technical report, Purdue University.

Gross, W., Wuttke, S. and Middelmann, W., 2015. Transformation of hyperspectral data to improve classification by mitigating nonlinear effects. In: Proceedings WHISPERS.

Hanneke, S., 2014. Theory of active learning. Technical report, Private.

Haraké, L., Schilling, H., Blohm, C., Hillemann, M., Lenz, A., Becker, M., Keskin, G. and Middelmann, W., 2016. Concept for an airborne real-time isr system with multi-sensor $3 \mathrm{~d}$ data acquisition. In: Optical Engineering + Applications, SPIE Proceedings, SPIE, p. 998709.

Hasanzadeh, M. and Kasaei, S., 2010. A multispectral image segmentation method using size-weighted fuzzy clustering and membership connectedness. IEEE Geoscience and Remote Sensing Letters 7(3), pp. 520-524.

Huo, L.-Z., Tang, P., Zhang, Z. and Tuia, D., 2015. Semisupervised classification of remote sensing images with hierarchical spatial similarity. IEEE Geoscience and Remote Sensing Letters 12(1), pp. 150-154.

Kääriäinen, M., 2006. Active learning in the non-realizable case. In: Algorithmic Learning Theory: Proceedings, Lecture Notes in Computer Science, Vol. 4264, pp. 63-77.

Kashef, R. and Kamel, M. S., 2009. Enhanced bisecting -means clustering using intermediate cooperation. Pattern Recognition 42(11), pp. 2557-2569.

Krempl, G., Kottke, D. and Lemaire, V., 2015. Optimised probabilistic active learning (opal). Machine Learning 100(2-3), pp. 449-476.

Lee, S., 2004. Efficient multistage approach for unsupervised image classification. In: International Geoscience and Remote Sensing Symposium: Proceedings, pp. 1581-1584.

Lee, S. and Crawford, M. M., 2004. Hierarchical clustering approach for unsupervised image classification of hyperspectral data. In: International Geoscience and Remote Sensing Symposium: Proceedings, pp. 941-944.
Lee, S. and Crawford, M. M., 2005. Unsupervised multistage image classification using hierarchical clustering with a bayesian similarity measure. IEEE Transactions on Image Processing 14(3), pp. 312-320.

Liu, R., Wang, Y., Baba, T., Masumoto, D. and Nagata, S., 2008. Svm-based active feedback in image retrieval using clustering and unlabeled data. Pattern Recognition 41(8), pp. 2645-2655.

Marcal, A. R. S. and Castro, L., 2005. Hierarchical clustering of multispectral images using combined spectral and spatial criteria. In: International Geoscience and Remote Sensing Symposium: Proceedings, Vol. 2, pp. 59-63.

Munoz-Mari, J., Tuia, D. and Camps-Valls, G., 2012. Semisupervised classification of remote sensing images with active queries. IEEE Transactions on Geoscience and Remote Sensing 50(10), pp. 3751-3763.

Pasolli, E. and Melgani, F., 2010. Active learning methods for electrocardiographic signal classification. IEEE transactions on information technology in biomedicine : a publication of the IEEE Engineering in Medicine and Biology Society 14(6), pp. 1405-1416.

Patra, S. and Bruzzone, L., 2011. A fast cluster-assumption based active-learning technique for classification of remote sensing images. IEEE Transactions on Geoscience and Remote Sensing 49(5), pp. 1617-1626.

Schindler, K., 2012. An overview and comparison of smooth labeling methods for land-cover classification. IEEE Transactions on Geoscience and Remote Sensing 50(11), pp. 4534-4545.

Senthilnath, J., Omkar, S. N., Mani, V., Diwakar, P. G. and Shenoy B, A., 2012. Hierarchical clustering algorithm for land cover mapping using satellite images. IEEE Journal of Selected Topics in Applied Earth Observations and Remote Sensing 5(3), pp. 762-768.

Settles, B., 2012. Active Learning. Synthesis Lectures on Artificial Intelligence and Machine Learning, Vol. 6, Morgan \& Claypool Publishers.

Tuia, D., Muñoz-Marí, J. and Camps-Valls, G., 2012. Remote sensing image segmentation by active queries. Pattern Recognition 45(6), pp. 2180-2192.

Tuia, D., Volpi, M., Copa, L., Kanevski, M. F. and Munoz-Mari, J., 2011. A survey of active learning algorithms for supervised remote sensing image classification. IEEE Journal of Selected Topics in Signal Processing 5(3), pp. 606-617.

Wassenberg, J., Middelmann, W. and Sanders, P., 2009. An efficient parallel algorithm for graph-based image segmentation. In: Computer Analysis of Images and Patterns: Proceedings, Springer Berlin Heidelberg, Berlin, Heidelberg, pp. 1003-1010.

Wuttke, S., Middelmann, W. and Stilla, U., 2015. Concept for a compound analysis in active learning for remote sensing. In: The International Archives of the Photogrammetry, Remote Sensing and Spatial Information Sciences, Vol. XL-3/W2, pp. 273-279.

Zhang, D., Wang, F., Shi, Z. and Zhang, C., 2008. Localized content based image retrieval by multiple instance active learning. In: International Conference on Image Processing. 